\title{
A Narrow QRS Complex Tachycardia: What is the Mechanism?
}

\author{
FRED MORADY, M.D.
}

From the Division of Cardiology, Department of Internal Medicine, University of Michigan Medical Center, Ann Arbor, Michigan

\section{Case Presentation}

A 65-year-old woman underwent an electrophysiologic procedure because of a 20 -year history of paroxysmal supraventricular tachycardia. The baseline sinus cycle length was $800 \mathrm{msec}$, with an atrial-His interval (AH) of $100 \mathrm{msec}$ and a His-ventricular interval (HV) of $40 \mathrm{msec}$. There was evidence of dual AV nodal pathways, but no inducible tachycardia. During infusion of isoproterenol, the sinus cycle length shortened to 460 to $500 \mathrm{msec}$, with an $\mathrm{AH}$ of $80 \mathrm{msec}$ and an $\mathrm{HV}$ of $40 \mathrm{msec}$. Atrial pacing at a cycle length of $360 \mathrm{msec}$ induced a supraventricular tachycardia

This manuscript was processed by a guest editor.

J Cardiovasc Electrophysiol, Vol. 12, pp. 1199-1201, October 2001.

Address for correspondence: Fred Morady, M.D., Division of Cardiology, Department of Internal Medicine, University of Michigan Medical Center, 1500 East Medical Center Drive, Ann Arbor, MI 49109-0022. Fax: 734936-7026; E-mail: fmorady@umich.edu that had a cycle length of 460 to $500 \mathrm{msec}$ (Fig. 1). The response to atrial pacing at a cycle length of $410 \mathrm{msec}$ during tachycardia is shown in Figure 2. A single ventricular extrastimulus introduced during tachycardia when the His bundle was refractory had no effect on the tachycardia. Double ventricular extrastimuli reliably restored sinus tachycardia with an AH interval of $80 \mathrm{msec}$ (Fig. 3). What is the mechanism of this tachycardia?

\section{Commentary}

The observation that atrial activation during tachycardia usually was coincident with ventricular activation reliably rules out orthodromic AV reentrant tachycardia using an accessory pathway. By exclusion, the tachycardia is likely to be arising in either the atrium or the AV junction. Atrial pacing during the tachycardia demonstrated that it was entrainable (Fig. 2), thereby ruling out an automatic junctional tachycardia and leaving an atrial tachycardia or AV nodal reentry as the most likely mechanism.

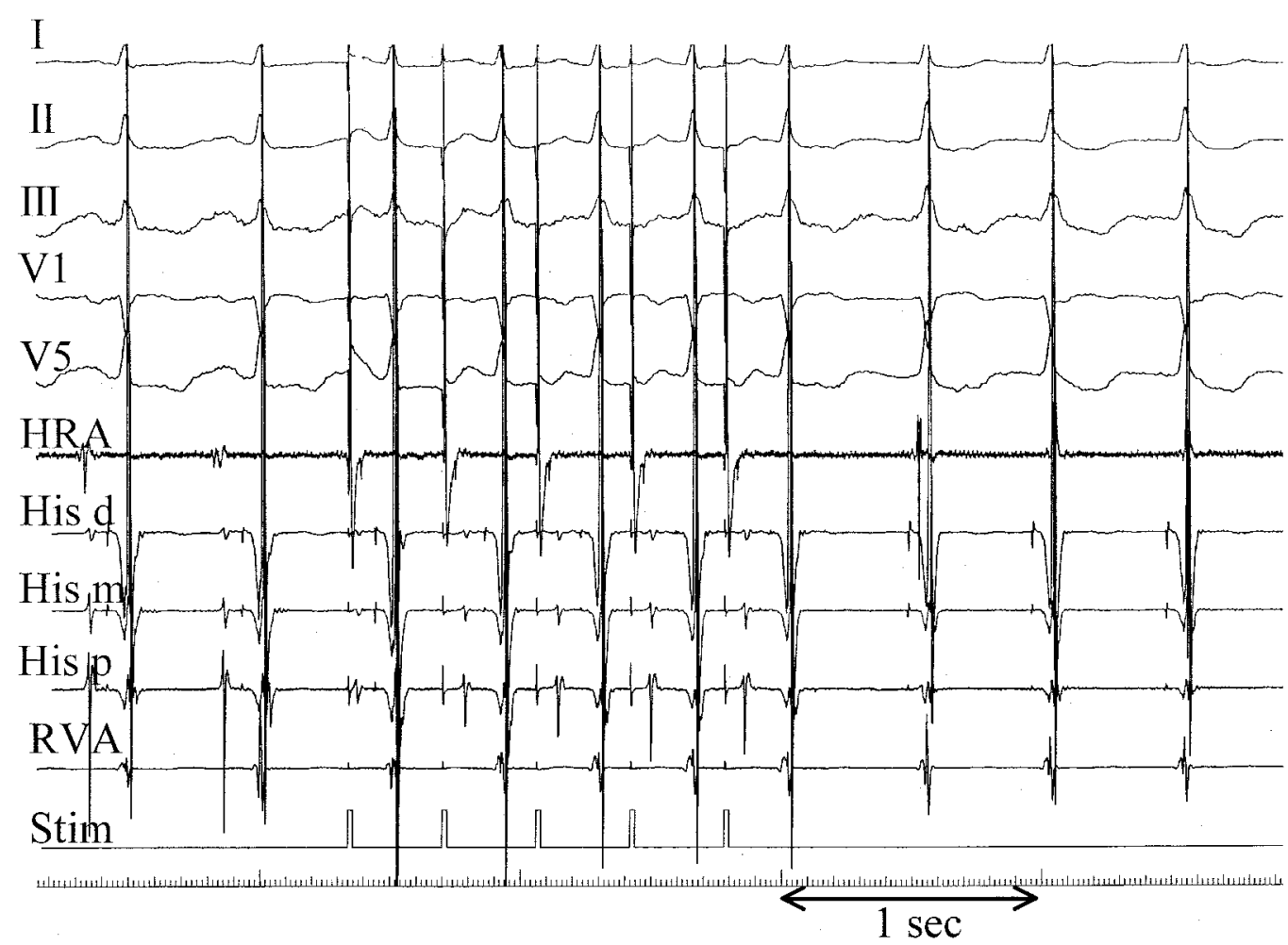

Figure 1. Induction of tachycardia, cycle length 480 to $500 \mathrm{msec}$, by atrial pacing at a cycle length of 360 msec during infusion of isoproterenol. This type of response to atrial pacing was reproducible. The sinus cycle length before atrial pacing is 500 msec. $d=$ distal; HRA $=$ high right atrial electrogram; $m=$ mid; $p=$ proximal; $R V A=$ right ventricular apex electrogram; Stim $=$ stimulus. 


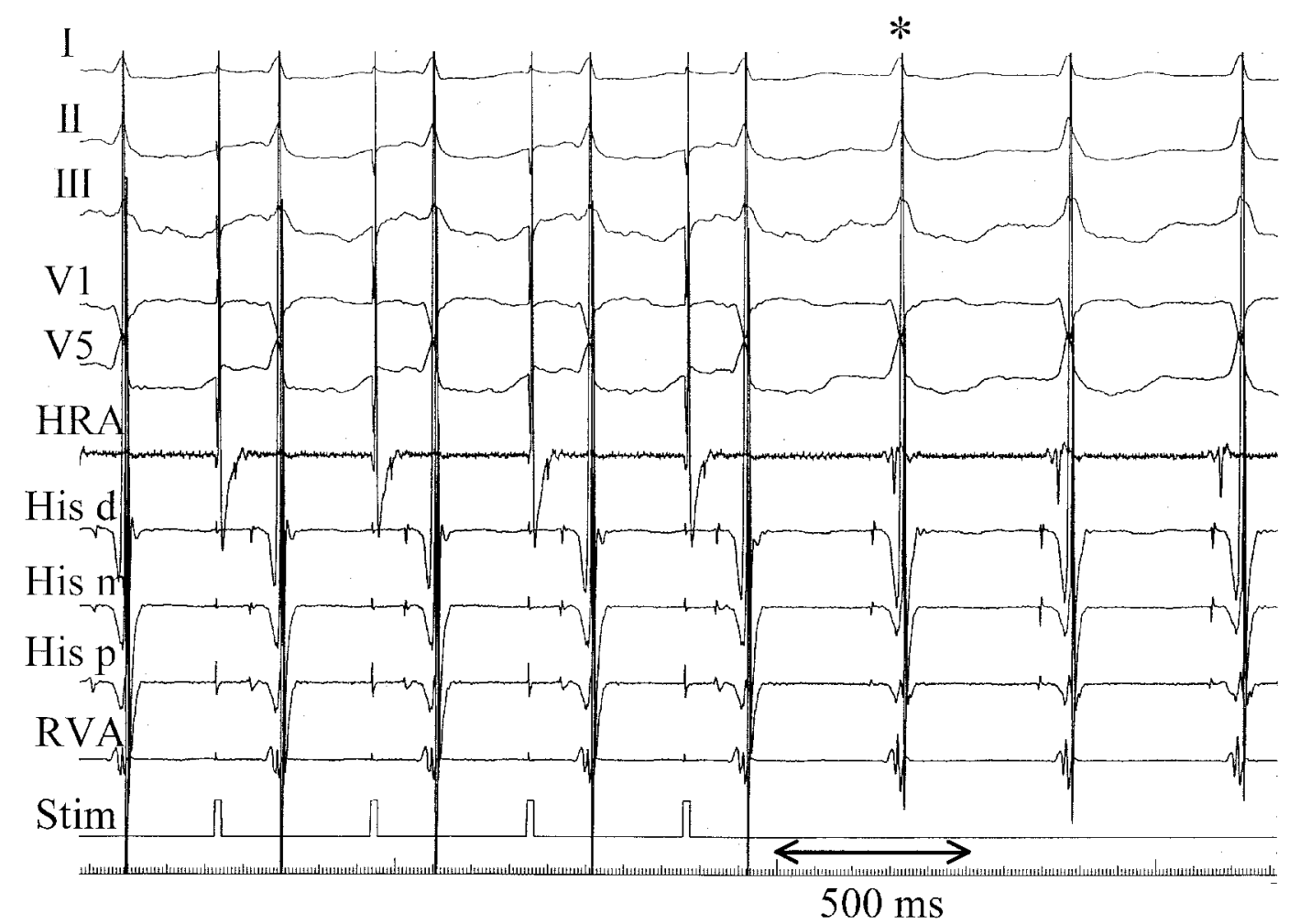

Figure 2. Response to atrial pacing at a cycle length of $410 \mathrm{msec}$ during tachycardia. Note that the QRS complex marked with an asterisk was advanced by the last pacing stimulus. Abbreviations as in Figure 1.

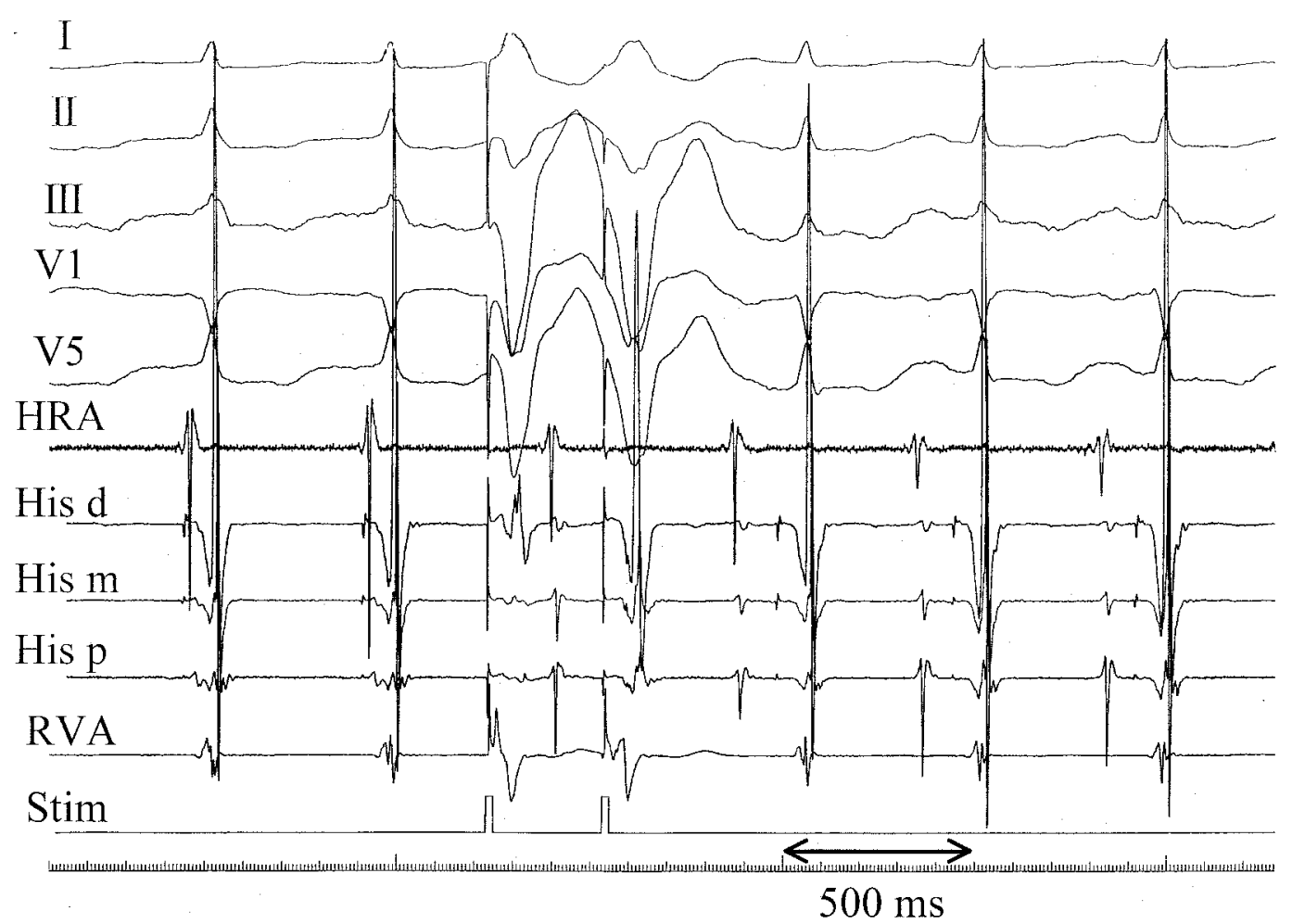

Figure 3. Response to two ventricular extrastimuli. Isoproterenol was being infused, and the cycle length before and after introduction of the two ventricular extrastimuli was constant at $500 \mathrm{msec}$. The AH interval changes from 460 to $80 \mathrm{msec}$. Abbreviations as in Figure 1. 
At first glance, the simultaneous atrial and ventricular activation during tachycardia appears compatible with AV nodal reentrant tachycardia. The induction of tachycardia shown in Figure 1 could be explained by a "double fire," in which the last pacing stimulus results in conduction through both a fast and slow AV nodal pathway. Recovery of fast pathway excitability by the time the wave of depolarization had traversed the slow pathway would allow retrograde conduction through the fast pathway and induction of $\mathrm{AV}$ nodal reentrant tachycardia.

However, there is an aspect of this tachycardia that cannot be explained by conventional AV nodal reentry: the atrial activation sequence during tachycardia is not concentric, but instead appears to be identical to the atrial activation sequence during sinus tachycardia. Note that in Figures 1 and 2, during tachycardia, there is no evidence of a septal atrial electrogram preceding the high right atrial electrogram, an observation that is not compatible with conventional AV nodal reentrant tachycardia. The fact that high right atrial activation precedes low septal atrial activation is confirmed in Figure 3, when atrial activation occurs a bit earlier than ventricular activation, making the "high-low" atrial activation sequence more apparent. Also noteworthy is the observation that the tachycardia cycle length is the same as the cycle length during sinus tachycardia.

These observations are compatible with simultaneous sinus tachycardia and AV nodal reentrant tachycardia. However, during multiple inductions of the tachycardia, there was very little variability in the AV relationship, making isorhythmic double tachycardias unlikely. One possible explanation for the relatively fixed AV relationship during tachycardia may be that sinus tachycardia was constantly entraining an AV nodal reentrant tachycardia. This mechanism also would explain why the cycle lengths during the two tachycardias were the same.

However, there is another possibility that may be as likely as AV nodal reentrant tachycardia that is entrained by sinus tachycardia. The tachycardia may simply be sinus tachycardia with simultaneous atrial and ventricular activation attributable to conduction down a slow AV nodal pathway. The response to ventricular pacing in Figure 3 is compatible with either termination of $\mathrm{AV}$ nodal reentry or a shift in anterograde conduction from the slow to fast AV nodal pathway.

This tachycardia raises an interesting question of semantics. If this were sinus tachycardia with conduction down the slow pathway, perpetuation of conduction to the ventricle through the slow pathway would require repeated retrograde concealed penetration of the fast pathway, rendering the fast pathway unavailable for anterograde conduction. From a functional viewpoint, this is precisely what occurs during entrainment of $\mathrm{AV}$ nodal reentrant tachycardia by atrial pacing (or, in this case, by sinus tachycardia).

Irrespective of the semantic uncertainty, it was clear that the patient's symptomatic tachycardia was attributable to simultaneous atrial and ventricular activation when anterograde conduction occurred through the slow pathway. Therefore, slow pathway ablation was performed. The long $\mathrm{AH}$ tachycardia was no longer inducible, and the patient has experienced no further episodes of symptomatic tachycardia during 6-month follow-up. 